\title{
Peculiar features of silicate materials curing
}

\author{
Yulia Sidorenko ${ }^{1 *}$, Alexander Guryanov ${ }^{1}$, and Vyacheslav Kozlov ${ }^{1}$ \\ ${ }^{1}$ Samara State Technical University, Academy of Civil Engineering and Architecture, \\ 194 Molodogvardeyskaya str., 443001, Samara, Russia
}

\begin{abstract}
The papers on contact-condensing (non-autoclave) curing of silicate materials point at the expediency of technology development. Nonautoclave silicate products of M $100 \ldots 300$ grades can be used in low-rise and individual housing construction. The complexity of practical implementation of the technology associated with the processes occurring in the field of highly dispersed phases requires research on micro capsulation of hydro silicate binder, in particular, the maintenance of its properties in technological redistribution. High surface energy of newgrowth causes particle clustering. It is desirable to obtain aggregates characterized by the lowest energy of their total intercrystallite interaction. Main mechanism of directed structure formation at the boundaries of the sections of the given binder is ultradisperse particles with a high adsorption capacity and adhesive ability, the main purpose of which is to strengthen the contact zone. The novelty of the proposed solutions lies in the fact that contact formation zone is presented as a multilayer system having different rheological characteristics. The study and description of contactcondensing processes occurring in unstable hydrosilicate systems as well as the sol-gel technologies are the important aspects in the foundations development of modern material science, the creation of materials and new generation products.
\end{abstract}

\section{Introduction}

The main costs in the construction materials production are associated with the following energy-intensive redistribution: grinding and crushing, heat treatment of raw materials and semi-finished products [1-5]. That is why, it is important to save fuel, energy and raw materials resources, particularly, in the production of silicate, lime-siliceous, cementcontaining materials and products.

Therefore, it is especially recommended to reduce the energy costs for raw materials grinding by introducing surfactants; secondary, use of waste energy in autoclaving; exclusion from the classical scheme of silicate brick production some redistributions (for example, ensiling) or introduction of new ones (additional mixture homogenization) to reduce the processing time of the raw material batch.

Parametric optimization of some technological indicators connected with their reduction (pressing pressure, duration of autoclave processing, etc.) is also recommended $[1,2]$.

\footnotetext{
* Corresponding author: $\underline{\text { sm-samgasa } @ \text { mail.ru }}$
} 
A perspective of energy-saving technologies is also the production of piece contactcondensing (non-autoclave) lime-siliceous (silicate) products.

The production of low-basic, unstable calcium hydro silicates $\left(\begin{array}{llll}0.8 & \ldots & 1.5\end{array}\right)$ $\mathrm{CaO} \cdot \mathrm{SiO} 2 \cdot \mathrm{nH}_{2} \mathrm{O}$ is carried out in an isothermal reactor, a periodic type crystallizer (processing temperature of the unstable mixture is $\sim 95^{\circ} \mathrm{C}$ ), then the binder and quartz filler are mixed until they acquire the molding moisture, at increased pressure $(40 \ldots 60 \mathrm{MPa})$ and followed by drying.

Due to contact-condensing processes waterproof raw blocks having $12 \ldots 15 \mathrm{MPa}$ are formed; after the drying (at $100 \ldots 200^{\circ} \mathrm{C}$ ), their strength increases approximately 1.5 times due to the completion of crystallization processes, which makes it possible to use nonautoclave silicate products M $100 \ldots 300$ grade in low-rise construction [1].

\section{Materials and Methods}

In the course of the work, raw materials caracterised as non-deficient local and common for many regions of the country raw materials were used; preparation of calcium hydrosilicates in the crystallizer is significantly different from autoclave technology.

The processes of dissolution of the initial components and the crystallization of newgrowth occur at high carrier phase rates (sharply increasing mass and heat transfer on the surfaces) and are accompanied by crushing, abrasion, and particle aggregation. High surface energy of newgrowth causes particle clustering.

From an operational point of view, it is desirable to obtain finally from the crystallizer aggregates with the lowest energy of their total intercrystallite interaction.

To eliminate the listed technological difficulties, it is proposed to use microencapsulation of an unstable hydrosilicate binder; this will improve the stability of its characteristics the quality at the stage of pressing raw blocks [3].

This field is a new one in terms of a scientific approach to solving practical problems of this kind, to creating a theoretical and practical basis for the production of microencapsulated compound silicate binders.

In the process of pressing, the incompletely saturated capillary-porous system undergoes strong structural changes both at macro- and microlevels.

A system of drains and sources is formed, which play a major role in the redistribution of the binder along the system, and the boundary being impenetrable for the liquid-solid phase and partially penetrable for the gas phase; thus, the formation of a water-resistant raw block frame (at the macrolevel) is regarded as the process of formation of phase contacts in the structure in the most stressed sites of the film phases [1].

When the percolation threshold is reached, the concentration of such contacts forms an infinite power percolation cluster, resulting in a sudden change in the strength of raw blocks properties [1].

At the microlevel, as a result of the motion of a heterogeneous multiphase medium through a convergent channel, gradients of tangential stresses and velocities are formed.

Under their action, the particles of the solid phase are rearranged increasing their density.

The transition is accompanied by the flow of liquid from the gaps, its destructuring and transition to the drainage area.

The role of drains will then be played by smaller peripheral unfilled capillaries that are filled with areas that are not completely filled with liquid and have a compressible gas part [1].

The heat released during pressing promotes an additional water outflow from the grid and an increase in the effect of the constraint of the system. 


\section{Results}

Hereby, as a result of the occurring mechanical fluid syneresis and the rearrangement of aggregates, the volume content of the highly dispersed component of the solid phase gradually increases along the length [1].

When small distances between particles are reached under the influence of the concentration factor, the wedging forces and the gradual particle transition towards the constrained state, and then towards the nearest potential pit take place.

The fact that the achievement area of a solid phase reaches critical concentration corresponds to the arising percolation condensation front, which divides the contact zone into two parts: the zone of the dynamic membrane and the zone of the capillary-porous body. The percolation zone gradually shifts to the bulk phase.

In the process of percolation, the solid phases of unreacted particles act a filler in the microsystem and do not create phase contacts [1].

When the pressing pressure is up to 50-60 $\mathrm{MPa}$, incomplete particle coalescence is performed at the level of colloidal particles of the gel mass, being the most active in the energetically. In the process of hydrothermal preparation of calc-siliceous mixture, large crystals of hydrosilicates formed before do not participate in contact-condensing processes, but act as an active micropores filler.

The gel matrix acts as a matrix of the binder and on its basis under the action of deformations, a power frame of the raw block is created. This is realized with the help of partial overlapping of loose clusters in the elastic points of the periodic gel grid.

There is also a more dense packing of the sol particle part, which were not included into the gel grid.

When the concentration of aggregates is equal to the critical one, an infinite percolation graph (cluster) is created over the overlapping spheres, which should be identified with thearising of phase irreversible contacts in the most energetic overlapping cluster areas, i.e. with the power frame of raw material [1].

This type of mechanism for creating a power frame presupposes the presence of sufficient supply of a gel component acting as a contact-condensing properties carrier in the compressible system, which is expended both for the large crystals enveloping and the filling of the cells remainng in the system [1].

For example, when cement concrete hardens under pressure, arising from the hydration of the clinker particle the gel layer is quite tightly bound with the particle surface and the possibility of its displacement from the surface is rather limited [1].

The remaining free water found in the bulk areas, during the further hydration feeds this gel layer and thereby creates a permanent wedging pressure, which excludes the particles transition towards the constrained energy level. Given that the density of the solid phase of the gel layer decreases towards the periphery, one should expect the introduction (overlapping) of such grids.

This overlap will grow with increasing of pressing energy. In the overlapping zone, a higher concentration of the solid phase will be observed, but it is highly likely that this concentration will not be sufficient for overcoming of the particles of the wedging barrier and creatjng phase contacts, since there is no kinetic component of the particles. Similar conditions will be possible when free water quit to supple gel.

Drying of the gel zone of the grain by water evaporating creates free volumes in it and increases the probability their filling; the role of external pressure in the process of creating strength being increased [1]. However, in connection with further increase in the limiting shear stress, the probability of such a transition will decrease.

There is a highly filled unstable phase between the contacted grains in the volumetric area is, which is less tightly bound to the structural elements, and which, under stresses up 
to the the critical ones $\left(\tau>\tau_{0}\right)$, is squeezed into the cavity of the necks, where conditions for the percolation phase transition are created.

The increment of potential energy is used to translate it into kinetic one, which will repack the particles and increase their concentration [1].

If the initial mixture is prepared according to the scheme of separate technology, the effect of pressing pressure will be more evident.

\section{Discussion}

A characteristic feature of given mixtures is the highly developed interface between individual phases and minerals, which requires an increase in strength between the individual elements of the structure.

The main mechanism of directed structure formation at the boundaries of the sections of these binders is ultradisperse particles with a high adsorption, adhesion capacity, which are to strengthen the contact zone (one of the most important structural units of multicomponent binders).

Microencapsulation of unstable hydrosilicate binder makes it possible to increase the stability of its characteristics at the pressing raw blocks stage, to ensure the stability of the technological process, and the quality of the products in whole.

The system of interest is characterized by the complicated topological network of capillaries, their diameter and length, as well as filling of cavities with phases. Furthermore, self-arrangement phenomena with threshold transitions occur in the system [1]. The pressing process of multicomponent systems is accompanied by structural and phase transitions: small portions of new hydrated calcium silicates appear, the binder breaks into free liquid and solid phase, solid unstable phase condenses, etc. Some of these transitions play a secondary role in contact-condensation hardening, while structural transitions in the binder are of primary importance. As load is applied, the binder is distributed through the system of pores interconnected by capillaries.

Redistribution of the binder causes the occurrence of gas blocks in capillaries, thus defining the spectrum of intergranular areas by the strength of their distribution. Interaction of local cluster structures of the binder leads to appearance of larger clusters and, ultimately, to appearance of an infinite cluster by the binder and its reaching the area boundary. Ensuring the strength of the semi-finished product is viewed as interconnection of two cluster structures of different physical levels: the binder as clusters of flow distribution through the system of capillaries and force bonds between the contacting areas of structural elements [1,2].

The study and description of contact-condensing processes occurring in unstable hydrosilicate systems as well as the sol-gel technologies are the important aspects in the foundations development of modern material science and the creation of materials and products of a new generation. [6-11].

The further researches can be based on the information submitted to the formation of structure and properties of the cement-containing materials (cellular concretes, light, heavy, etc.).

\section{Conclusion}

The studies of contact and condensing (non-autoclave) hardening of silicate materials point out the reasonability of developing this technology.

Non-autoclave silica products (M 100...300) grade can be used in low-rise buildings (typical and individual design).

Key technological operations in the production technology of non-autoclaved silicate products include preparation of the binder, mixing the binder and the filler, pressing the 
products and their further drying (temperature from $70^{\circ} \mathrm{C}$ ). The binder is prepared by mixed grinding of the original components proportioned in the ratio of 5:7. Further steam treatment of silica-lime binders is performed at the temperature of $90 \ldots 100^{\circ} \mathrm{C}$ for 2 hours, water-solid ratio is maintained at $5 \ldots 8$. The prepared mixture is pressed at $40 \ldots 60 \mathrm{MPa}$ to form the product.

After drying, compressive strength of the pressed products is $15 \ldots 20 \mathrm{MPa}$; water absorption percentage is $\sim 10 \%$, frost resistance is - 35 cycles (meets the requirements of the Russian Standard GOST 379-2015 Silicate Bricks, Stones, Blocks and Partition Blocks. General Specifications [1, 2, 11]. Analysis of the research shows [1, 2] that preferred parameters for practical application are: pressure $54 \ldots 56 \mathrm{MPa}$, pressing time $25-27 \mathrm{sec}$.

The difficulty of practical implementation of the technology connected with the processes which take place in the sphere of highly dispersed phases requires research, in particular in the area of hydrosilicate binder microencapsulation and maintaining its properties on technological limits.

The high surface energy of new formations causes the clusterization of particles. The novelty of the suggested solutions lies in the representation of contact formation zone as a multilayer system with different rheological properties. The dynamics of the formation of capillary-porous partition between structure-forming elements is a combination of interconnected submodels.

Studies of mutual coordination and kinetics of skeleton formation of construction composite materials, modeling its probability structure are among the least explored areas of contemporary Materials Science [11-14].

\section{References}

1. Y.V. Sidorenko, Modelling of contact-condensation processes of low-basic calcium hydrosilicates hardening: Cand. Diss. (Tech. Sci.) (SGASU, Samara, 2003)

2. Y.V. Sidorenko, S. F. Korenkova, Multi-Level Synergetic Approach to Formation of the Hardening Mechanism of Contact-Condensate Systems of The Silicate Type (SSUACE, Samara, 2005)

3. V.E. Romanenkov, E.E. Petyushik, Physical and chemical bases of hydration powder media hardening (Belarusian Navuka, Minsk, 2012)

4. A.I. Gusev, A.A. Rempel, Nanocrystalline materials (Physmathlit, Moscow, 2001)

5. V.I. Vereshchagin, L.P. Rikhvanov, Yu.S. Sarkisov, Yu.F. Asoskov, A.P. Smirnov, Tomsk Polytechnic University Proceedings, 315 (3), 12 (2009)

6. H. Haken, Secrets of Nature. Synergetics: the doctrine about cooperation (Institute of Computer Science, Moscow-Izhevsk, 2003)

7. I.V. Melikhov, Physical and chemical evolution of the solid substance (BINOM, Laboratory of knowledge, Moscow, 2006)

8. L.I. Loitsiansky, Fluid and Gas Mechanics (Drofa, Moscow, 2003)

9. V.I. Roldugin, Physics and chemistry of the surface (TCC INTELLIGENCE, Moscow, 2008)

10. P.V. Konarev, M.V. Petoukhov, V.V. Volkov, D.I. Svergun, J. Appl. Cryst., 39, 277 (2006)

11. Y.V. Sidorenko, S. F. Korenkova, A. M. Guryanov, MATEC 106, 03031 (2017)

12. E.V. Romanova, Solid State Phenom 871, 132 (2016)

13. E.V. Romanova, J. of Economy and entrepreneurship 9 (74), 984 (2016)

14. E. V. Romanova, MATEC 73, 07010 (2016) 